\title{
Influence of the mask magnification on imaging in hyper-NA lithography
}

\author{
Chun-Hung Lin \\ National Nano Device Laboratories, Hsinchu 300, Taiwan \\ Hsuen-Li Chen \\ Department of Materials Science and Engineering, National Taiwan University, Taipei 106, Taiwan \\ Fu-Hsiang Ko \\ Institute of Nanotechnology, National Chiao Tung University, Hsinchu 300, Taiwan \\ Received October 3, 2006; revised December 22, 2006; accepted December 30, 2006; \\ posted January 10, 2007 (Doc. ID 75706); published May 9, 2007

\begin{abstract}
Argon fluoride laser (ArF) lithography using immersion technology has the potential to extend the application of optical lithography to $45 \mathrm{~nm}$ half-pitch and possibly beyond. By keeping the same $4 \times$ magnification factor, the dimensions of the structures on masks are becoming comparable to the exposure wavelength or even smaller. The polarization effect induced by mask features is, however, an issue. The introduction of a larger mask magnification should be strongly considered when poor diffraction efficiencies from subwavelength mask features and the resulting image degradation would be encountered in hyper-NA lithography. The dependence of the diffraction efficiencies on mask pitch and illuminating angle are evaluated. The near-field intensity and phase distributions from the mask are calculated. The imaging performance of $4 \times$ and $8 \times$ masks for the sub$45 \mathrm{~nm}$ node are explored. A rigorous coupled-wave analysis is developed and employed to analyze the optical diffraction from the 3D topographic periodic features. (C) 2007 Optical Society of America
\end{abstract}

OCIS codes: $110.5220,050.1960$.

\section{INTRODUCTION}

Argon fluoride laser (ArF) lithography using immersion technology has the potential to extend the applicability of optical lithography to $45 \mathrm{~nm}$ half-pitch and possibly beyond. ${ }^{1}$ Several techniques, such as higher-index materials, ${ }^{2-4}$ polarization illumination, ${ }^{5,6}$ and the multiple exposure technique, ${ }^{6-8}$ are further developed to extend $193 \mathrm{~nm}$ immersion lithography beyond $45 \mathrm{~nm}$ halfpitch. However, by keeping the same $4 \times$ magnification factor, the dimensions of the structures on masks are becoming comparable to the exposure wavelength or even smaller. The polarization effect induced by the mask features is, however, an issue. ${ }^{9-12}$ The improved resolution capabilities of the optical systems also increase the sensitivity of these systems to the mask defects. Therefore, the defect printability at the $4 \times$ mask will be a severe problem. ${ }^{13}$ The mask cost would increase significantly because of the stringent mask critical dimension (CD) control and the use of the complicated optical proximity correction or attendant features. Moreover, the traditionally used Kirchhoff approximation is no longer valid to describe the light diffraction from the mask. A more complicated rigorous electromagnetic modeling method should be employed in designing the resolution enhancement masks or in modeling the optical proximity behavior. This would result in a longer calculating cycle time. The change to a higher mask magnification factor could relax the above-mentioned severe situations. ${ }^{6,14,15}$ The cost of the lens system could be reduced due to the reduced exposure field size. However, the lower throughput due to a smaller field size would then be the major concern. The introduction of a larger mask magnification should be strongly considered when the poor diffraction efficiencies from the subwavelength mask features and the resulting image degradation would be encountered in hyper-NA lithography.

In this work, the rigorous coupled-wave analysis $(\mathrm{RCWA})^{16-18}$ is developed and employed to analyze the optical diffraction from the $3 \mathrm{D}$ topographic periodic features. Section 2 presents our approach of mask diffraction analysis with the RCWA algorithm and the subsequent high-NA vector imaging analysis. In Section 3, the dependences of the diffraction efficiencies of an attenuated phase-shifting mask (attPSM) on feature pitches and illuminating angles are illustrated. The near-field intensity and phase distributions from the mask are calculated. The imaging performance of $4 \times$ and $8 \times$ masks for the sub-45 nm node are compared. Finally, we conclude in Section 4 with a summary.

\section{ANALYSIS}

Traditionally, the Kirchhoff boundary condition has been widely used in aerial image simulations. ${ }^{19}$ The mask is assumed to be infinitely thin and the transmitted electric 
field is assumed to be a perfect step function with a constant phase shift. However, when the feature sizes on the mask are of the order of the wavelength or even smaller, this assumption is not accurate and a rigorous electromagnetic method, ${ }^{16-18,20,21}$ such as RCWA, finitedifference time domain (FDTD) method, finite-element method, or waveguide method, should be employed to take into account the 3D topographic effects on the mask. Among the above approaches, the FDTD and RCWA are the most widely used methods to analyze the light diffraction from the 3D topography on masks. The FDTD method discretizes Maxwell's equations in both time and space in a straightforward manner and solves the scattering problem by simulating the field evolution through time until the time-harmonic steady-state solution is reached. The perfect matched layer (PML) $\operatorname{method}^{22}$ is adopted to restrict the simulated problems in a finite computational domain. The incident waves are applied with the total and scattered field formulation. ${ }^{23}$ The speed and accuracy of the FDTD method depend on the space and time discretization, the total simulating time period, and the PML parameters. Although the FDTD method is capable of simulating arbitrary $2 \mathrm{D}$ or $3 \mathrm{D}$ geometries, considerably larger computing resources in terms of computing time and memory are required.

The RCWA method describes the propagating fields inside the mask with periodic structures by a plane wave expansion. Maxwell's equations are then converted into a system of linear ordinary differential equations by applying spatial Fourier expansions of the field and the permittivity. The speed and accuracy of the RCWA method depend on the number of retained orders. ${ }^{17,18}$ The advantage of using the RCWA is that the transmitted field from the mask has been expressed in terms of the spatial Fourier expansion, which can be directly employed in the following aerial image analysis. Moreover, when simulating $2 \mathrm{D}$ periodic structures such as contact-hole patterns, computing efficiency can be improved considerably more by applying the symmetry properties of the grating diffraction problem. ${ }^{24}$

Figure 1 illustrates the geometry of the RCWA configuration for the mask. The whole structure can be divided into three regions: the incident region (region I), the grating region (region II), and the exit region (region III). The incident normalized electric field can be expressed in the form

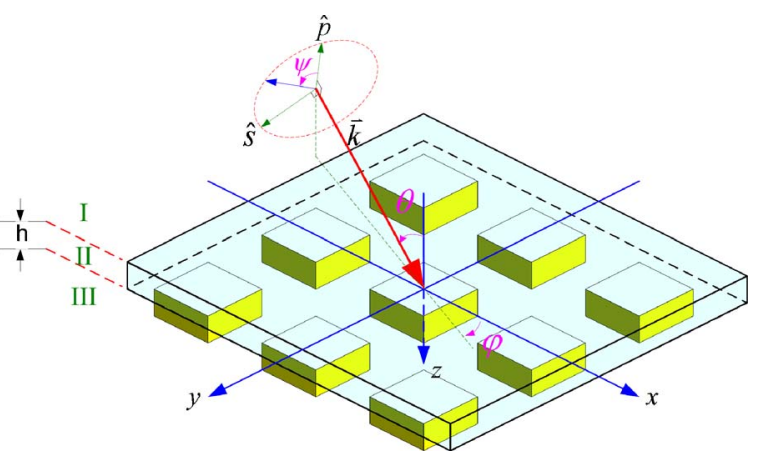

Fig. 1. (Color online) Geometry of the RCWA configuration for the mask.

$$
\mathbf{E}_{i n c}=\hat{u} \exp \left[-j\left(k_{i n c, x} x+k_{i n c, y} y+k_{i n c, z} z\right)\right],
$$

where $\hat{u}$ is the unit polarization vector, $k_{\text {inc, } x}$ $=k_{0} n_{I} \sin \theta \cos \varphi, \quad k_{\text {inc }, y}=k_{0} n_{I} \sin \theta \sin \varphi, \quad k_{\text {inc }, z}=k_{0} n_{I} \cos \theta$, $k_{0}=2 \pi / \lambda$, and $n_{I}$ is the refractive index of the incident region. The total electric fields in the external regions (I, III) can be expressed as

$$
\begin{gathered}
\mathbf{E}_{\mathrm{I}}=\mathbf{E}_{i n c}+\sum_{m, n} \mathbf{R}_{m n} \exp \left[-j\left(k_{x m} x+k_{y n} y-k_{\mathrm{I} z, m n} z\right)\right], \\
\mathbf{E}_{\mathrm{III}}=\sum_{m, n} \mathbf{T}_{m n} \exp \left\{-j\left[k_{x m} x+k_{y n} y+k_{\mathrm{III} z, m n}(z-h)\right]\right\},
\end{gathered}
$$

where $\mathbf{R}_{m n}$ and $\mathbf{T}_{m n}$ are the complex amplitudes of $(m, n)$ th reflected and transmitted orders, respectively. The wave vector components $k_{x m}$ and $k_{y n}$ can be determined from the Floquet conditions and are given by

$$
\begin{array}{r}
k_{x m}=k_{i n c, x}+m\left(\frac{2 \pi}{\Re \Lambda_{x}}\right), \\
k_{y n}=k_{i n c, y}+n\left(\frac{2 \pi}{\Re \Lambda_{y}}\right), \\
k_{x m}^{2}+k_{y n}^{2}+k_{l z, m n}^{2}=\left(k_{0} n_{l}\right)^{2}, \quad l=\mathrm{I}, \mathrm{III},
\end{array}
$$

where $\mathfrak{R}$ is the reduction factor of the projection system. For example, $\Re$ is equal to 4 for the $4 \times$ reduction; $\Lambda_{x}$ and $\Lambda_{y}$ are the periods of the grating along the $x$ and $y$ directions in the wafer scale. $n_{\mathrm{I}}$ and $n_{\mathrm{III}}$ are the refractive indices of the incident and exit regions.

The magnetic fields in the external regions can be obtained from Maxwell's equation

$$
\mathbf{H}=-\frac{1}{j \omega \mu_{0}} \nabla \times \mathbf{E},
$$

where $\mu_{0}$ is the permeability of free space and $\omega$ is the angular optical frequency. In the grating region, the periodic permittivity can be expressed in the Fourier expansion:

$$
\varepsilon_{\mathrm{II}}(x, y)=\sum_{g, h} \varepsilon_{g h} \exp \left[j k_{0}\left(\frac{\lambda}{\Re \Lambda_{x}} g x+\frac{\lambda}{\Re \Lambda_{y}} h y\right)\right] .
$$

The electric and magnetic fields in the grating region can be expressed with a Fourier expansion in terms of the space-harmonic fields,

$$
\begin{aligned}
& \mathbf{E}_{\mathrm{II}}=\sum_{m, n} \mathbf{S}_{m n}(z) \exp \left[-j\left(k_{x m} x+k_{y n} y\right)\right], \\
& \mathbf{H}_{\mathrm{II}}=-j \sqrt{\frac{\varepsilon_{0}}{\mu_{0}}} \sum_{m, n} \mathbf{U}_{m n}(z) \exp \left[-j\left(k_{x m} x+k_{y n} y\right)\right],
\end{aligned}
$$

where $\varepsilon_{0}$ and $\mu_{0}$ are the permittivity and permeability of free space, respectively. $\mathbf{S}_{m n}$ and $\mathbf{U}_{m n}$ are the complex amplitudes of the $(m, n)$ th space-harmonic fields. Finally, by applying Maxwell's equation in the grating region and matching the boundary conditions at the interface of the three regions, the unknown amplitudes $\mathbf{R}_{m n}$ and $\mathbf{T}_{n m}$ of the diffracted waves can be determined. 
The near-field distribution of the periodic mask can thus be treated as the complex amplitude distribution of the object in the imaging system and is rewritten directly from Eq. (2),

$$
\mathbf{E}_{\mathrm{O}}(x, y)=\sum_{m, n} \mathbf{T}_{m n} \exp \left[j k_{0} n_{\mathrm{III}}\left(\alpha_{m} x+\beta_{n} y\right)\right],
$$

where $\left(\alpha_{m}, \beta_{n}\right)=\left(k_{x m}, k_{y n}\right) /\left(k_{0} n_{\mathrm{III}}\right)$ and $\alpha_{m}^{2}+\beta_{n}^{2}+\gamma_{m n}^{2}=1$; $\left[\alpha_{m}, \beta_{n}, \gamma_{m n}\right]$ are the unit propagation vectors of the diffracted beams from the mask. $n_{\mathrm{III}}$ is the refractive index of the exit region from the mask, which is air and equal to 1 in current optical lithography. By considering the reduction factor $\mathfrak{R}$ and image inversion, the mask object can be further expressed as

$$
\begin{aligned}
\mathbf{E}_{G}(\tilde{x}, \tilde{y}) & =\mathfrak{R} \mathbf{E}_{\mathrm{O}}(-\mathfrak{R} \tilde{x},-\mathfrak{R} \tilde{y}) \\
& =\Re \sum_{m, n} \mathbf{T}_{m n} \exp \left[j k_{0} n_{\text {fluid }}\left(\alpha_{m}^{\prime} \tilde{x}+\beta_{n}^{\prime} \tilde{y}\right)\right],
\end{aligned}
$$

where $(\tilde{x}, \tilde{y})=-(x, y) / \Re$ are the reduced coordinates in the object plane. $n_{\text {fluid }}$ is the refractive index of the immersion fluid. $\left[\alpha_{m}^{\prime}, \beta_{n}^{\prime}, \gamma_{m n}^{\prime}\right]$ are the unit propagation vectors of the diffracted light propagating into the exit pupil of the optical system and are related with the vectors $\left[\alpha_{m}, \beta_{n}, \gamma_{m n}\right]$ from the entrance pupil by

$$
\begin{aligned}
\left(\alpha_{m}^{\prime}, \beta_{n}^{\prime}\right) & =\frac{\Re}{n_{\text {fluid }}}\left(\alpha_{m}, \beta_{n}\right), \\
\alpha_{m}^{\prime 2}+{\beta_{n}^{\prime}}^{2}+\gamma_{m n}^{\prime}{ }^{2} & =1 .
\end{aligned}
$$

To calculate the corresponding image, the mask object, the pupil function of the optical system, the influence of polarization in image formation, and the resist thin-film effects should be taken into account. The final image distribution can be expressed as ${ }^{9}$

$$
\begin{aligned}
\mathbf{E}_{\mathrm{I}}\left(x^{\prime}, y^{\prime}\right) & =\left[\begin{array}{c}
E_{I, x} \\
E_{I, y} \\
E_{I, z}
\end{array}\right] \\
& =\mathcal{F}^{-1}\left\{\mathbf{F}\left(\alpha^{\prime}, \beta^{\prime} ; z^{\prime}\right) \mathbf{P}\left(\alpha^{\prime}, \beta^{\prime}\right) \mathbf{E}_{G}\left(\alpha^{\prime}, \beta^{\prime}\right) H\left(\alpha^{\prime}, \beta^{\prime}\right)\right\},
\end{aligned}
$$

where $\mathbf{E}_{G}\left(\alpha^{\prime}, \beta^{\prime}\right)$ is the spatial spectrum of the mask object obtained with the Fourier transform operation. The complex amplitudes $\mathbf{T}_{m n}$ of $(m, n)$ th transmitted orders determined from the RCWA approach can be treated as the amplitudes of the $(m, n)$ th spatial components of the mask directly:

$$
\begin{aligned}
\mathbf{E}_{G}\left(\alpha^{\prime}, \beta^{\prime}\right)=\left[\begin{array}{c}
E_{G, x} \\
E_{G, y} \\
E_{G, z}
\end{array}\right] & =\mathcal{F}\left\{\mathbf{E}_{G}(\tilde{x}, \tilde{y})\right\} \\
& =\Re \sum_{m, n} \mathbf{T}_{m n} \delta\left(\alpha^{\prime}-\alpha_{m}^{\prime}\right) \delta\left(\beta^{\prime}-\beta_{n}^{\prime}\right) .
\end{aligned}
$$

The pupil function $H$ describes the conditions of the individual spatial components inside the optical system. Higher frequency components of the diffracted beams are filtered out by the pupil and the transmitted frequency components then interfere at the wafer surface to form the aerial image. Assuming that the optical system has no polarization dependency in our analysis, the pupil function $H$ can be scalar in the following form:

$$
H\left(\alpha^{\prime}, \beta^{\prime}\right)=0 \text { for } \sqrt{\alpha^{\prime 2}+\beta^{\prime 2}}>\frac{N A}{n_{\text {fluid }}} .
$$

In a high-NA optical system, the diffracted beams propagate at very large angles relative to the optical axis and then come into interference at the image plane. Therefore, the influence of polarization effects on the image formation should also be taken into account. The polarization matrix $\mathbf{P}$ decomposes the $x, y$, and $z$ components of the field vector into its TE and TM components at the image plane.

$$
\mathbf{P}\left(\alpha^{\prime}, \beta^{\prime}\right)=\left[\begin{array}{ccc}
\frac{\beta^{\prime 2}}{1-\gamma^{\prime 2}} & \frac{-\alpha^{\prime} \beta^{\prime}}{1-\gamma^{\prime 2}} & 0 \\
\frac{\alpha^{\prime 2} \gamma^{\prime} \gamma}{1-\gamma^{\prime 2}} & \frac{\alpha^{\prime} \beta^{\prime} \gamma^{\prime} \gamma}{1-\gamma^{\prime 2}} & -\gamma^{\prime} \alpha \\
\frac{-\alpha^{\prime} \beta^{\prime}}{1-\gamma^{\prime 2}} & \frac{\alpha^{\prime 2}}{1-\gamma^{\prime 2}} & 0 \\
\frac{\alpha^{\prime} \beta^{\prime} \gamma^{\prime} \gamma}{1-\gamma^{\prime 2}} & \frac{\beta^{\prime 2} \gamma^{\prime} \gamma}{1-\gamma^{\prime 2}} & -\gamma^{\prime} \beta \\
-\alpha^{\prime} \gamma & -\beta^{\prime} \gamma & \alpha^{\prime} \alpha+\beta^{\prime} \beta
\end{array}\right] .
$$

If the light passes through the entrance pupil with a relatively small angle to the optical axis, i.e., $\gamma \cong 1$ and $\alpha^{2}$ $+\beta^{2} \cong 0$, the polarization matrix $\mathbf{P}$ is then reduced to the form in Refs. 25 and 26. Finally, if one needs to take account of the thin-film effects of the resist stack, the thinfilm matrix F derived by Flagello ${ }^{26}$ et al. should also be considered in Eq. (10).

\section{RESULTS}

Attenuated phase shifting mask (AttPSM) is a wellestablished resolution enhancement technique in $193 \mathrm{~nm}$ lithography and has been widely used for device manufacturing. The process latitude is improved as compared with that of the binary mask and its design and manufacturing is much easier than alternating PSM. ${ }^{27}$ Therefore, the case of commonly used MoSi-based attPSM is demonstrated here. The phase absorber material is assumed to be MoSiON with the optical constant $(2.343,0.586)$ (Ref. 12 ), and will be used in all the following simulations. The thickness of MoSiON is $72 \mathrm{~nm}$ to provide a $180^{\circ}$ phase shift and $6.4 \%$ transmission. Figure 2 shows the simulated diffraction efficiencies of the attPSM with 1:1 line and pace patterns. The mask magnification factors of 4 $\times$ and $8 \times$ were compared and the dimensions were normalized to the wafer scale. In the Kirchhoff approximation, the diffraction efficiencies of an attPSM with line and space patterns can be written as 


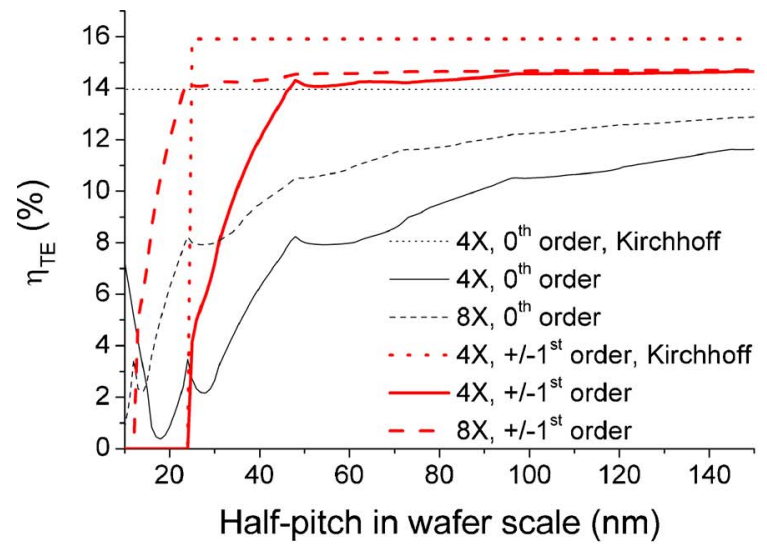

(a)

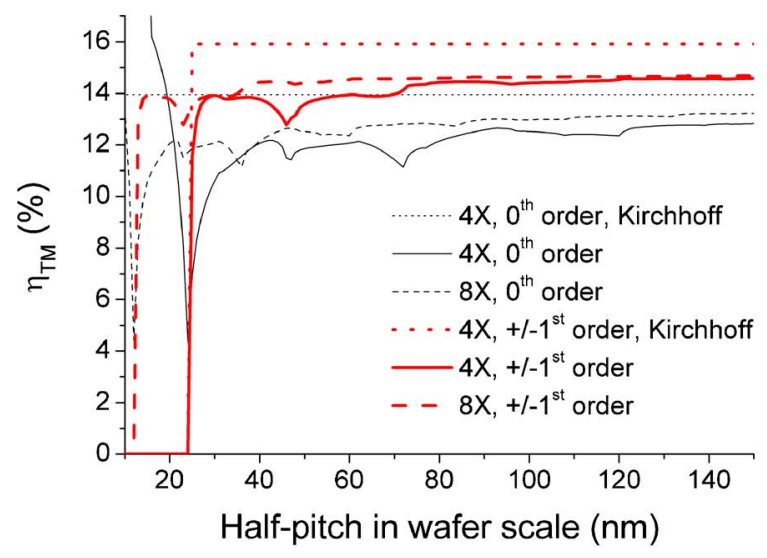

(b)

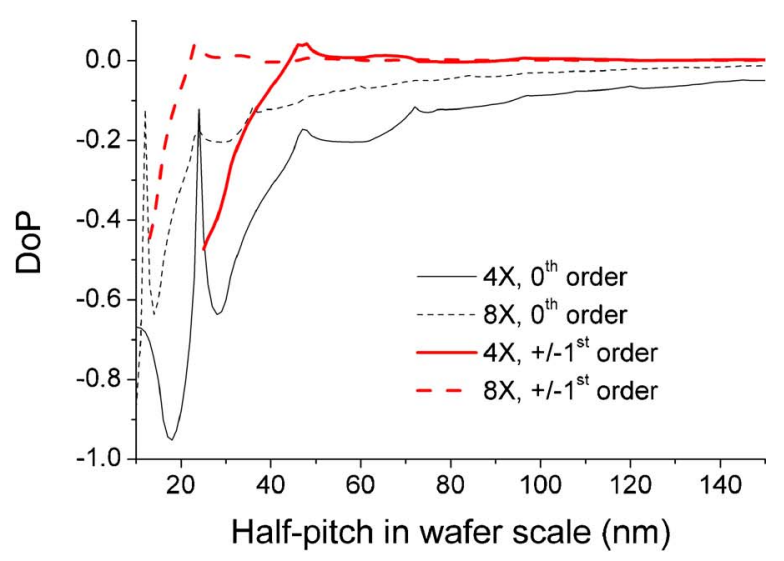

(c)

Fig. 2. (Color online) Diffraction efficiencies of the attPSM with 1:1 line and space patterns illuminated with TE- and TMpolarized light at normal incidence.

$$
\eta_{\text {Kirchhoff }}=\left|(1+\sqrt{T}) \frac{C D}{\Lambda} \operatorname{sinc}\left(m \frac{C D}{\Lambda}\right)-\sqrt{T} \operatorname{sinc}(m)\right|^{2},
$$

where $T$ is the transmission of the absorber, $C D$ is the linewidth of the opening region, $\Lambda$ is the pitch, and $m$ is the diffracted order of the transmitted light. With the Kirchhoff approximation, the diffraction efficiencies depend only on the transmission of the absorber and the ra- tio between linewidth and pitch. To take into account the 3D topography and the further optical properties of the absorber, the rigorous electromagnetic calculations should be applied. As shown in Fig. 2(a), there is an obvious turning point at the $48.25 \mathrm{~nm}$ half-pitch in the wafer scale, which is equal to the illuminating wavelength $193 \mathrm{~nm}$ on the $4 \times$ mask. When the patterns are smaller than the $48.25 \mathrm{~nm}$ half-pitch, the diffraction efficiencies of TE-polarized light decrease sharply. The diffraction efficiencies of the zeroth and \pm 1 st diffracted lights are $10.52 \%$ and $14.55 \%$, respectively, in the $100 \mathrm{~nm}$ half-pitch and those values decrease to $3.62 \%$ and $8.59 \%$ in the $32 \mathrm{~nm}$ half-pitch. For the $8 \times$ mask, the turning point is lowered to $24.125 \mathrm{~nm}$ half-pitch. The diffraction efficiencies of the zeroth and \pm 1 st diffracted lights can be kept to $8.20 \%$ and $14.26 \%$ in the $32 \mathrm{~nm}$ half-pitch and therefore, better contrast images can be formed. The diffraction ef-

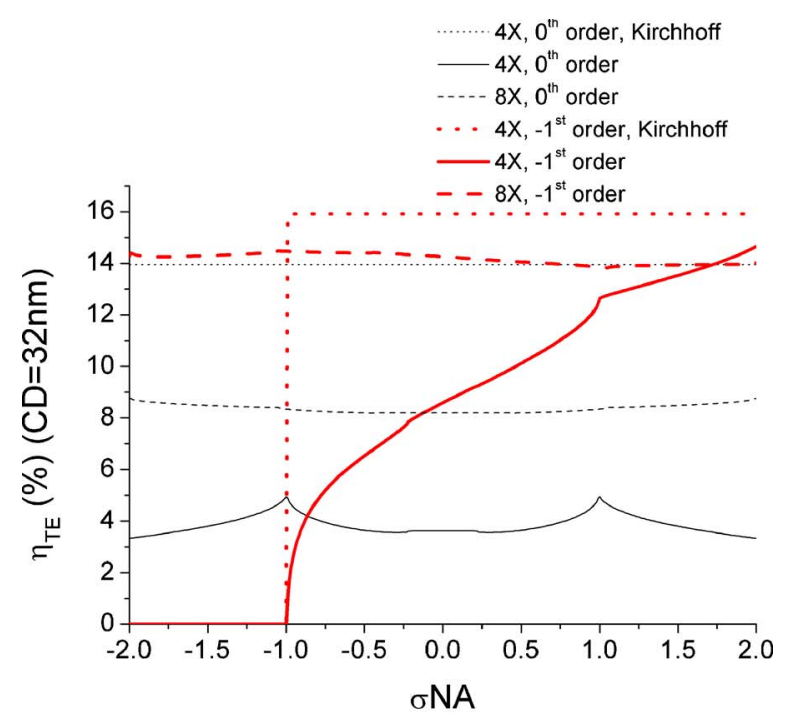

(a)

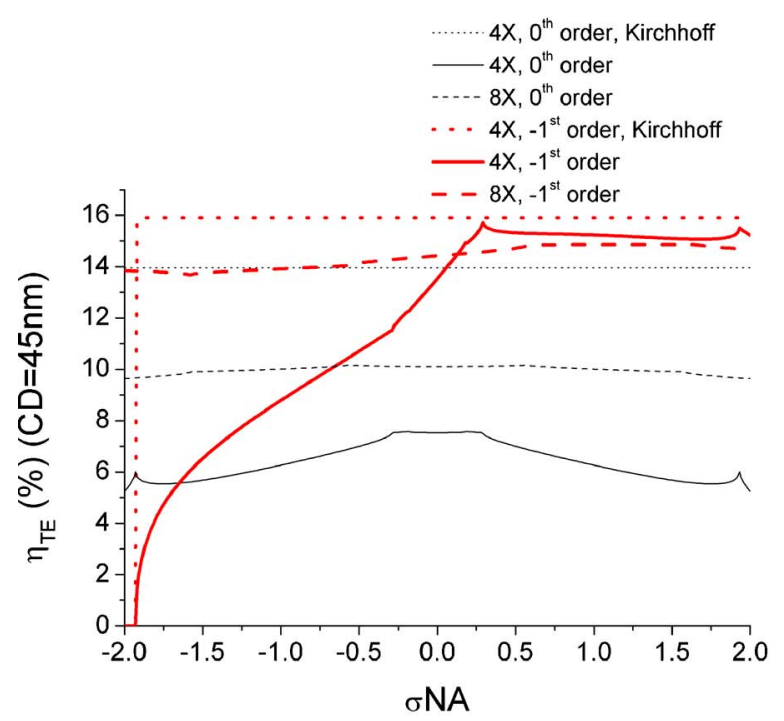

(b)

Fig. 3. (Color online) Diffraction efficiencies of the attPSM versus the illumination angle with TE-polarized light. The patterns are (a) 32 and (b) $45 \mathrm{~nm} \mathrm{1:1} \mathrm{line} \mathrm{and} \mathrm{space} \mathrm{in} \mathrm{the} \mathrm{wafer} \mathrm{scale.}$ 


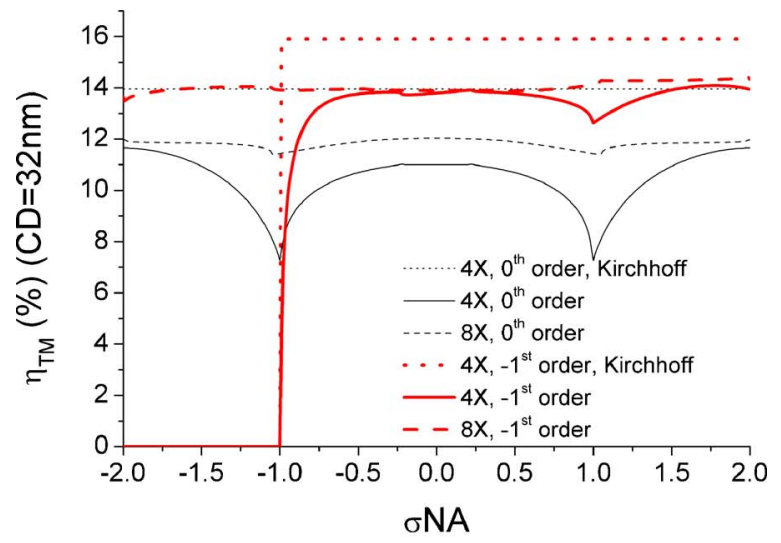

(a)

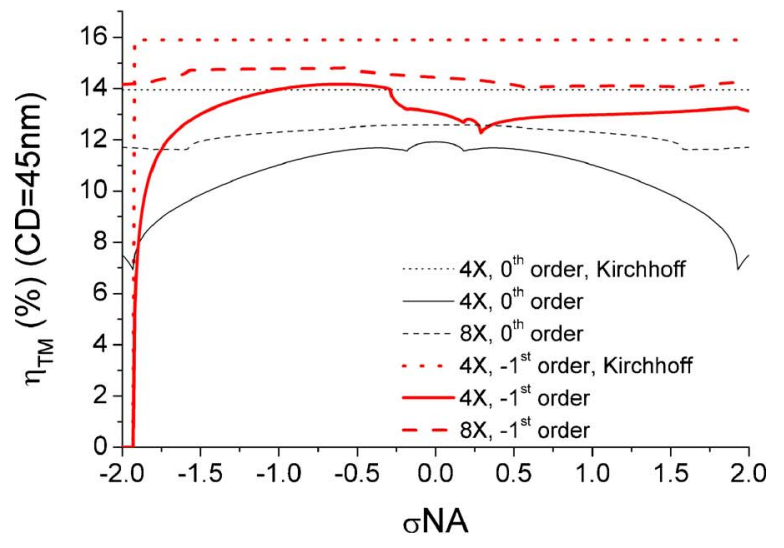

(b)

Fig. 4. (Color online) Diffraction efficiencies of the attPSM versus the illumination angle with TM-polarized light. The patterns are (a) 32 and (b) $45 \mathrm{~nm} \mathrm{1:1} \mathrm{line} \mathrm{and} \mathrm{space} \mathrm{in} \mathrm{the} \mathrm{wafer} \mathrm{scale.}$

ficiencies of the TM-polarized light were also simulated in Fig. 2(b). The diffraction efficiencies of the zeroth and \pm 1 st diffracted lights are $11.00 \%$ and $13.80 \%$, respectively, in the $32 \mathrm{~nm}$ half-pitch of the $4 \times$ mask. The diffraction efficiencies of the TM-polarized light are apparently better than those of TE-polarized light, which is similar to the behavior of a wire-grid polarizer ${ }^{28}$ and has been mentioned in Refs. 10 and 11. Figure 2(c) shows the mask polarizing effects in terms of the degree of polarization (DOP):

$$
\mathrm{DOP}=\frac{\eta_{\mathrm{TE}}-\eta_{\mathrm{TM}}}{\eta_{\mathrm{TE}}+\eta_{\mathrm{TM}}} .
$$

It is found that the $4 \times$ mask tends to polarize the transmitted light into the TM polarization state especially for a half-pitch smaller than $\lambda / 4$ in the wafer scale. For the $8 \times$ mask, the apparent polarizing phenomenon can be postponed to $\lambda / 8$.

Because of the off-axis illumination in the advanced lithography, the variation in the diffraction efficiencies with different illuminating angles was also evaluated. In the Kirchhoff approximation, the diffraction efficiencies are independent of the illuminating angles, which is valid for mask pitches much larger than the illuminating wavelength. However, when the mask pitch is smaller than the illuminating wavelength, the diffraction efficiencies vary significantly with the illuminating angle. As shown in Fig. 3(a), the TE zeroth diffraction efficiency of the $4 \times$ mask is $3.62 \%$ in the normal incidence and has a maximum value of $4.94 \%$ when $\sigma \mathrm{NA}$ is equal to \pm 1 . This is much smaller than $13.95 \%$ obtained from the Kirchhoff

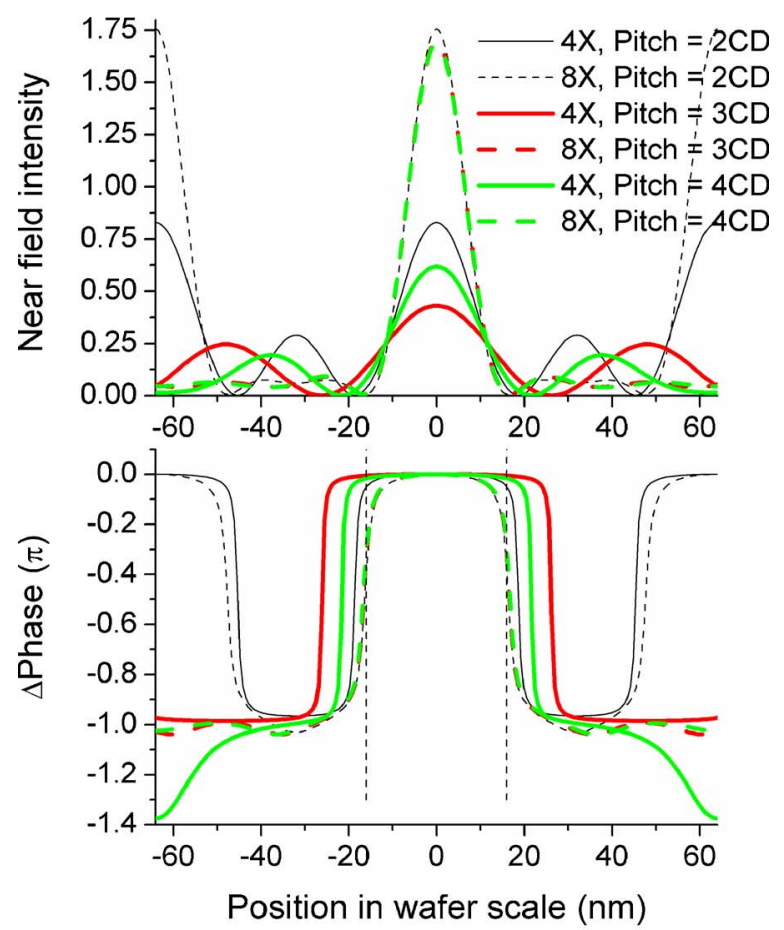

(a)

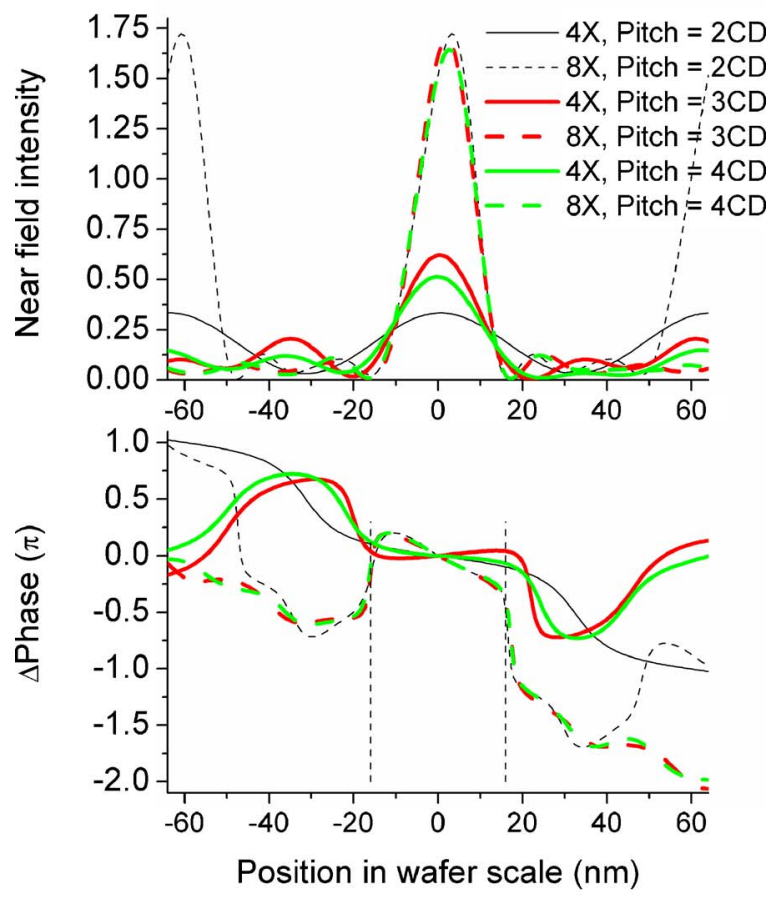

(b)

Fig. 5. (Color online) Near-field intensity and phase distributions from $32 \mathrm{~nm}$ line and space features in wafer scale. The illuminations are in (a) normal incidence and (b) off-axis with an angle corresponding to $\sigma \mathrm{NA}=1.47$. 

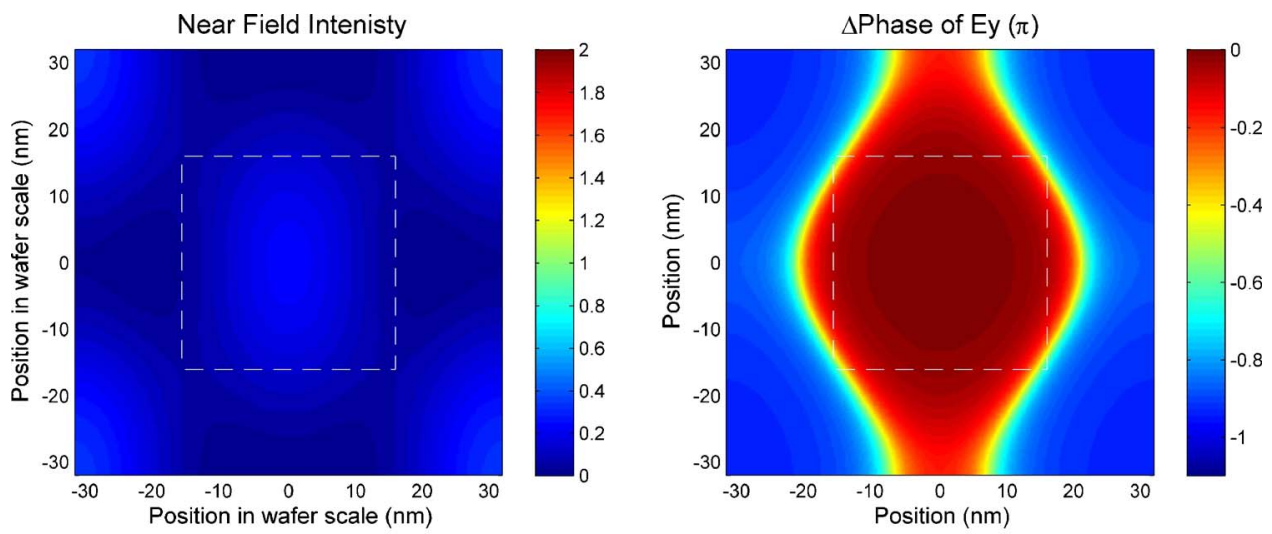

(a)
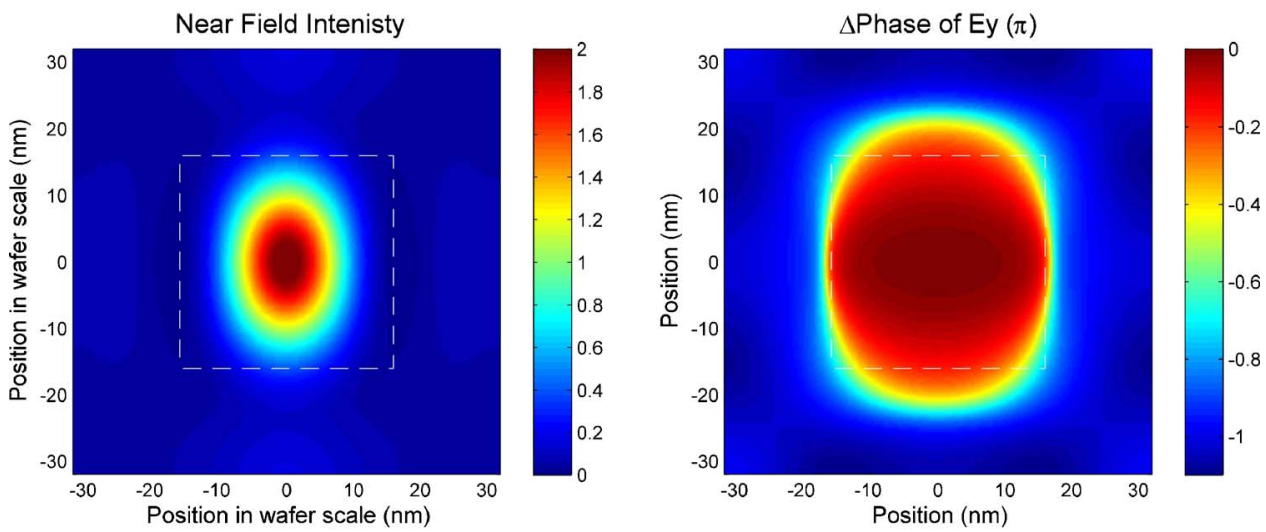

(b)

Fig. 6. (Color online) Near-field intensity and phase distributions of the (a) $4 \times$ and (b) $8 \times$ mask with $32 \mathrm{~nm}$ contact-hole pattern in $1: 1$ hole-to-space ratio.

approximation. The TE zeroth diffraction efficiency of the $8 \times$ mask varies from $8.19 \%$ to $8.76 \%$. The pattern is $32 \mathrm{~nm}$, equal line and space to the wafer scale. To include the mask magnification factor, the illuminating angle is defined in terms of $\sigma \times \mathrm{NA}$,

$$
\sigma \mathrm{NA}=\Re \sin \theta .
$$

The TE -1 st diffraction efficiency of the $4 \times$ mask is $8.59 \%$ in the case of normal incidence. The -1 st diffracted light vanishes when $\sigma \mathrm{NA} \leq-1$ and increases up to $12.65 \%$ when $\sigma \mathrm{NA}=1$. For the $8 \times$ mask, its -1 st diffraction efficiency varies from $13.82 \%$ to $14.48 \%$. The zeroth and -1 st diffraction efficiency in the $8 \times$ mask approach the Kirchhoff approximation and are almost independent of the illuminating angle. The case of $45 \mathrm{~nm}$ half-pitch was also simulated as shown in Fig. 3(b) for comparison. For $\sigma \mathrm{NA}$ larger than 0.2 , the diffraction efficiency of the -1 st diffracted light varies from $15.00 \%$ to $15.72 \%$, which is almost independent of the illuminating angle and approaches the Kirchhoff approximation. A $\sigma$ NA larger than 0.2 is usually found in the region of the operating condition with off-axis illumination. Figure 4(a) shows the diffraction efficiencies of the TM polarization. For the $4 \times$ mask, the TM zeroth diffraction efficiency is $11.00 \%$ for normal incidence and has a minimum value of.7.27\% when $\sigma \mathrm{NA}$ is equal to \pm 1 . The -1 st diffraction efficiency varies between $12.44 \%$ and $14.09 \%$ when $\sigma \mathrm{NA}$ is larger than -0.85 , which is much better than the case of TE polarization. When $\sigma \mathrm{NA}$ is smaller than -0.85 , the diffraction efficiency of the -1 st diffracted light drops quickly to zero which is similar to the behavior of the Kirchhoff approximation. From Eq. (3), the -1st diffracted light of the $4 \times$ mask turns to an evanescent wave when $\sigma \mathrm{NA}$ is smaller than -1 . Together with the simulation results in Figs. 2-4, the TM transmitted light from the $4 \times$ mask can maintain relatively good diffraction efficiencies down to around $30 \mathrm{~nm}$ half-pitch in the wafer scale. The diffraction efficiencies are not strongly dependent on the illuminating angle even in the case of $32 \mathrm{~nm}$ half-pitch with the $4 \times$ mask. However, the TE transmitted light is preferred in hyper-NA lithography. The image contrast under TM illumination is worse than that under the TE illumination because of the $\cos (2 \theta)$ factor in TM imaging.

$$
\begin{aligned}
& I_{\mathrm{TE}}(x)=A_{1}^{2}+A_{-1}^{2}+2 A_{1} A_{-1} \cos (2 k x \sin \theta), \\
& I_{\mathrm{TM}}(x)=A_{1}^{2}+A_{-1}^{2}+2 A_{1} A_{-1} \cos (2 k x \sin \theta) \cos (2 \theta),
\end{aligned}
$$

where $A_{1}, A_{-1}$ are the amplitudes of the two beams, $\theta$ is the incident angle in the imaging space, $k$ is equal to $2 \pi \mathrm{n} / \lambda$, and $n$ is the refractive index of the imaging space. Because of the better TE diffraction efficiencies from the 
$8 \times$ mask, the migration from $4 \times$ masks to $8 \times$ masks should be considered for the $32 \mathrm{~nm}$ half-pitch node.

To visualize the intensity and phase distributions after the exposing light passes through the 3D topographic features, the near-field distributions of the masks were calculated as shown in Fig. 5. The patterns are $32 \mathrm{~nm}$ CD in 64,96 , and $128 \mathrm{~nm}$ pitches in the wafer scale and were assumed to be illuminated with TE-polarized light. The cases of normal incidence and off-axis illumination with an angle corresponding to $\sigma \mathrm{NA}=1.47$ were simulated. For the $8 \times$ mask, the shapes of the near field intensity in the mask transmitting region are similar in all pitches regardless of the incident angle. There are $180^{\circ}$ phase differences between the mask transmitting and attenuating regions, which is characteristic of the attPSM. The positions of the phase drop between the transmitting and attenuating regions are situated at the pattern edges of the $8 \times$ mask. For the $4 \times$ mask, the image contrast of the near-field intensity is much poorer than those of the $8 \times$ mask. The positions of the phase drop between the transmitting and attenuating regions are deviated from the

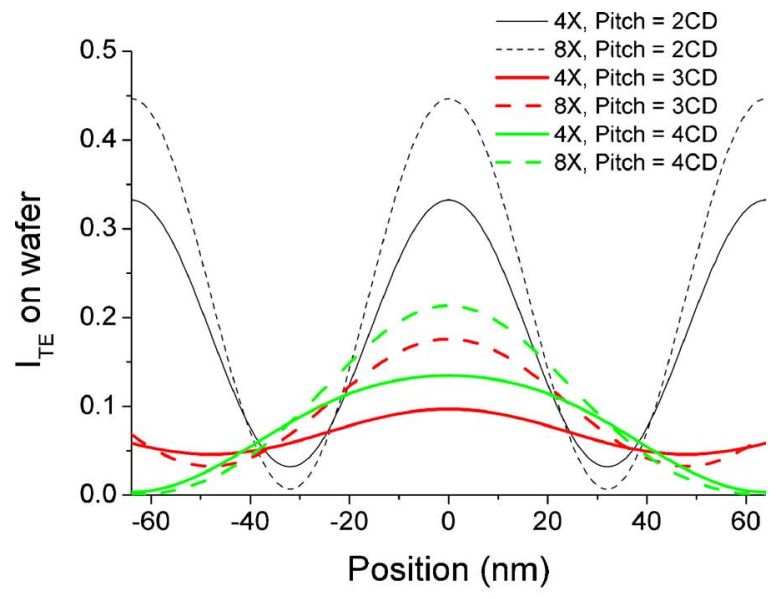

(a)

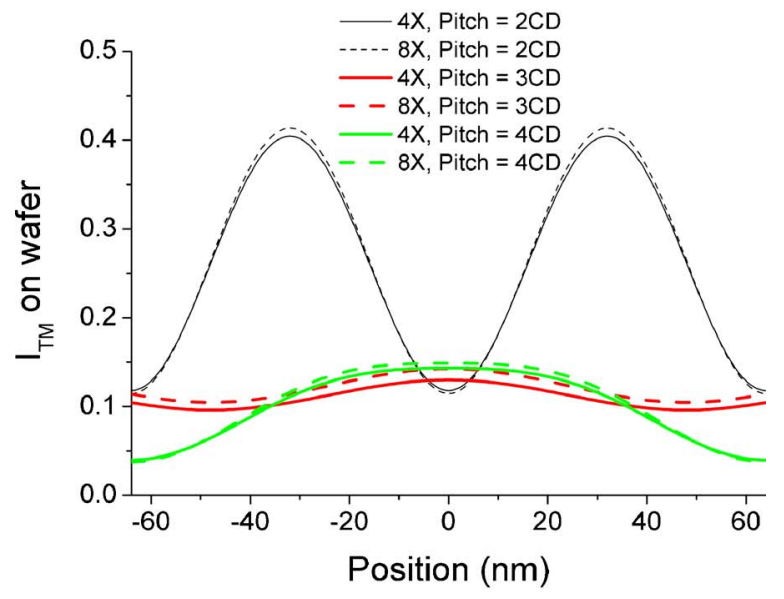

(b)

Fig. 7. (Color online) Aerial images of $32 \mathrm{~nm} \mathrm{CD}$ in the immersion fluid. The NA of the imaging system was assumed to be 1.55 with a high-index immersion fluid and lens material. Illumination is with (a) TE polarization and (b) TM polarization in the dipole configuration with $\sigma_{\text {center }}=0.9$ and $\sigma_{\text {radius }}=0$. pattern edges in the case of normal incidence. The phase distributions are worse and deviate far from the Kirchhoff assumption in the off-axis angle $\sigma \mathrm{NA}=1.47$. There are no distinct $180^{\circ}$ phase differences between the mask transmitting and attenuating regions for the $4 \times$ mask. The near-field intensities and phases of the contact-hole pattern were simulated as shown in Fig. 6. The mask CD is $32 \mathrm{~nm}$ in the wafer scale with a 1:1 hole-to-space ratio. The exposing light was assumed to be polarized in the direction of the $y$ axis with normal incidence. For the $8 \times$ mask, there is a strong peak in the hole center and the $180^{\circ}$ phase difference is clear between the mask transmitting and attenuating regions. However, for the $4 \times$ mask, the peak intensity is much smaller and is only $15.7 \%$ of the value of the $8 \times$ mask owing to the lower diffraction efficiencies in the subwavelength features. Moreover, there are strong sidelobes in the four corners for the $4 \times$ mask. The near-field phase distribution deviates from the pattern shape. In summary, the $8 \times$ mask shows a better near-field intensity and phase distribution both in the $1 \mathrm{D}$ and the $2 \mathrm{D}$ patterns.

Traditionally, in the aerial image calculation, shift invariance of the diffraction spectrum is assumed to simplify the simulation process. Only the diffraction spectrum with a normal incidence needs to be calculated. The diffraction spectrum for oblique incidence is approximated by shifting the diffraction spectrum of normal incidence in the direction of the incident light. However, from the above analyses, it was found that the diffraction spectrum will be strongly dependent on the illumination angle in hyper-NA lithography. Therefore, the shift invariance property is no longer valid and Abbé's approach should be used instead of Hopkins's approach in the following aerial image simulation. In Abbé's method, the illuminating source is discretized into mutually independent coherent point sources. The final aerial image is then obtained by an incoherent superposition of all the contributions from each point source. Figure 7 shows the aerial images in the immersion fluid. The resist thin-film effects are not considered in our analysis. The patterns are $32 \mathrm{~nm}$ CD in 64, 96 , and $128 \mathrm{~nm}$ pitches in the wafer scale. The NA of the imaging system was assumed to be 1.55 with the highindex immersion fluid and lens material. The illumination is in the dipole configuration with $\sigma_{\text {center }}=0.95$ and $\sigma_{\text {radius }}=0$. Both the TE and TM polarization were simulated. The solid curves are the aerial image from the $4 \times$ mask and the dashed curves are from the $8 \times$ mask. The images are normalized to the open frame intensity, which is the intensity of a clear mask without any feature. In the TE illumination, the aerial images of the $8 \times$ mask show a better image contrast owing to the higher diffraction efficiencies from the $8 \times$ mask. The reflection between the immersion fluid and the photoresist is not taken into account here. Because the reflectance increases with an increased incident angle, the image contrast will be worse than those shown in Fig. 7. In TM illumination, there are no significant differences between the aerial images of the $4 \times$ and $8 \times$ mask. However, the image contrasts are not good, which has been mentioned in the diffraction efficiency analysis. Furthermore, it is found that the intensity peak of the 1:1 line and space pattern under TM illumination is shifted one half-pitch in the lateral direction. 
This will happen when the incident angles are larger than $45^{\circ}$. From the Eq. (17), the $\cos (2 \theta)$ factor is negative when $\theta$ is larger than $45^{\circ}$.

\section{CONCLUSION}

In this study, the influence of mask magnification on lithographic imaging for the sub- $45 \mathrm{~nm}$ node was evaluated with RCWA. The TE and TM diffraction efficiencies from the $4 \times$ and $8 \times$ attPSM mask were compared. Although the diffraction efficiencies of the TM transmitted light from the $4 \times$ mask are not strongly dependent on the illuminating angle and are kept relatively high in the $32 \mathrm{~nm}$ half-pitch, the TM-polarized light is not preferred due to poor image contrast. For TE-polarized light, the diffraction efficiencies of the $4 \times$ mask decrease sharply from the $\lambda / 4$ half-pitch, i.e., $48.25 \mathrm{~nm}$. In the near-field distribution of the $4 \times$ mask for the $32 \mathrm{~nm} \mathrm{CD}$, the positions of the phase drop between the transmitting and attenuating regions deviate from the pattern edges in the case of normal incidence. The situation is worse in the case of off-axis illumination, $\sigma \mathrm{NA}=1.47$, and the phase distribution cannot be kept correctly. The introduction of higher mask magnification can relax these severe situations seen with the $4 \times$ mask. For the $8 \times$ mask, the obvious decrease in the TE diffraction efficiencies can be lowered to $\lambda / 8$ half-pitch, i.e., $24.125 \mathrm{~nm}$. The diffraction efficiencies can be almost independent of the illuminating angle. Distinct $180^{\circ}$ phase differences between the mask transmitting and attenuating regions are maintained. Therefore, better lithographic performance can be obtained by using the $8 \times$ mask. Furthermore, the mask cost and defect control problem can be reduced. If hyper-NA lithography with a high-index immersion fluid and lens material can be obtained, the migration from $4 \times$ to $8 \times$ masks should be strongly considered.

\section{ACKNOWLEDGMENTS}

This work was supported by National Science Council, Taiwan (grant 05095Z9000). The authors are grateful to the National Center for High-Performance Computing for the support of the computing facilities.

C.-H. Lin's e-mail address is chlin@mail.ndl.org.tw.

\section{REFERENCES}

1. These data are available at http://www.itrs.net/Links/ 2005ITRS/Home2005.htm.

2. R. H. French, H. Sewell, M. K. Yang, S. Peng, D. McCafferty, W. Qiu, R. C. Wheland, M. F. Lemon, L. Markoya, and M. K. Crawford, "Imaging of 32-nm 1:1 lines and spaces using 193-nm immersion interference lithography with second-generation immersion fluids to achieve a numerical aperture of 1.5 and a $\mathrm{k} 1$ of 0.25 ," J. Microlithogr., Microfabr., Microsyst. 4, 031103 (2005).

3. T. M. Bloomstein, M. F. Marchant, S. Deneault, D. E. Hardy, and M. Rothschild, "22-nm immersion interference lithography," Opt. Express 14, 6434-6443 (2006).

4. J. Zhou, Y. Fan, A. Bourov, and B. W. Smith, "Inorganic immersion fluids for ultrahigh numerical aperture $193 \mathrm{~nm}$ lithography," Appl. Opt. 45, 3077-3082 (2006).

5. M. McCallum, G. Fuller, and S. Owa, "From hyper NA to low NA,” Microelectron. Eng. 83, 667-671 (2006).
6. B. J. Lin, "The ending of optical lithography and the prospects of its successors," Microelectron. Eng. 83, 604-613 (2006).

7. T. B. Chiou, A. Chen, S. Hsu, M. Eurlings, and E. Hendrickx, "Development of automatic OPC treatment and layout decomposition for double dipole lithography for lowkl imaging," Proc. SPIE 5645, 21-31 (2005).

8. H. Nakamura, Y. Onishi, K. Sato, S. Tanaka, S. Mimotogi, $\mathrm{K}$. Hashimoto, and S. Inoue, "Contact hole formation by multiple exposure technique in ultralow k1 lithography," J. Microlithogr., Microfabr., Microsyst. 4, 023005 (2005).

9. D. Flagello, B. Geh, S. Hansen, and M. Totzeck, "Polarization effects associated with hyper-numericalaperture (>1) lithography," J. Microlithogr., Microfabr., Microsyst. 4, 031104 (2005).

10. A. Estroff, Y. Fan, A. Bourov, and B. Smith, "Mask-induced polarization effects at high numerical aperture," J. Microlithogr., Microfabr., Microsyst. 4, 031107 (2005).

11. A. Erdmann, "Mask modeling in the low k1 and ultrahigh NA regime: phase and polarization effects," Proc. SPIE 5835, 69-81 (2005).

12. M. H. Bennett, A. Grenville, S. D. Hector, S. R. Palmer, L. H. A. Leunissen, V. Philipsen, T. M. Bloomstein, D. E. Hardy, M. Rothschild, and J. N. Hilfiker, "Experimental measurements of diffraction for periodic patterns by $193 \mathrm{~nm}$ polarized radiation compared to rigorous EMF simulations," Proc. SPIE 5754, 599-610 (2005).

13. A. Erdmann, T. Graf, K. Bubke, I. Höllein, and S. Teuber, "Mask defect printing mechanisms for future lithography generations," Proc. SPIE 6154, 61541C (2006).

14. K. Takehisa, "Magnification factor should be $8 \times$ for the 45 nm node," Microlithogr. World 13, 10-25 (2004).

15. S. Hector, "Mask magnification considerations: $8 \times$ over 4 $\times$ ? Not yet," Microlithogr. World 13, 17-26 (2004).

16. M. G. Moharam and T. K. Gaylord, "Three-dimensional vector coupled-wave analysis of planar-grating diffraction," J. Opt. Soc. Am. 73, 1105-1112 (1983).

17. L. Li, "New formulation of the Fourier modal method for crossed surface-relief gratings," J. Opt. Soc. Am. A 14, 2758-2767 (1997)

18. C. H. Lin, H. L. Chen, W. C. Chao, C. I. Hsieh, and W. H. Chang, "Optical characterization of two-dimensional photonic crystals based on spectroscopic ellipsometry with rigorous coupled-wave analysis," Microelectron. Eng. 83, 1798-1804 (2006).

19. J. W. Goodman, Introduction to Fourier Optics, 2nd ed. (McGraw-Hill, 1996), pp. 44-45.

20. A. K. Wong and A. R. Neureuther, "Rigorous threedimensional time-domain finite-difference electromagnetic simulation for photolithographic applications," IEEE Trans. Semicond. Manuf. 8, 419-431 (1995).

21. S. Burger, R. Kohle, L. Zschiedrich, W. Gao, F. Schmidt, R. Marz, and C. Nolscher, "Benchmark of FEM, waveguide and FDTD algorithms for rigorous mask simulation," Proc. SPIE 5992, 599216 (2005).

22. J. P. Berenger, "Three-dimensional perfectly matched layer for the absorption of electromagnetic waves," J. Comput. Phys. 127, 363-379 (1996).

23. K. Umashankar and A. Taflove, "A novel method to analyze electromagnetic scattering of complex objects," IEEE Trans. Electromagn. Compat. 24, 397-405 (1982).

24. C. Zhou and L. Li, "Formulation of the Fourier modal method for symmetric crossed gratings in symmetric mountings," J. Opt. A 6, 43-50 (2004).

25. M. Mansuripur, "Distribution of light at and near the focus of high-numerical-aperture objectives," J. Opt. Soc. Am. A 3, 2086-2093 (1986).

26. D. G. Flagello, T. Milster, and A. E. Rosenbluth, "Theory of high-NA imaging in homogeneous thin films," J. Opt. Soc. Am. A 13, 53-64 (1996).

27. A. K. Wong, Resolution Enhancement Techniques in Optical Lithography (SPIE, 2001), pp. 139-151.

28. E. Hecht, Optics, 2nd ed. (Addison-Wesley, 1987), p. 279. 